DOI: $10.3724 /$ SP.J.1218.2011.00174

\title{
基于内容理解的单幅静态街景图像深度估计
}

\author{
李 乐, 张茂军, 熊志辉, 徐玮 \\ (国防科学技术大学信息系统与管理学院, 湖南 长沙 410073)
}

\begin{abstract}
摘 要: 提出一种通过分析理解单幅街景图像内的景物构图关系实现图像深度估计的方法. 该方法首先对单幅 街景图像分块并提取图像块自身的特征以及邻域联合特征, 通过机器学习的方法根据图像块的特征识别图像中的各 类景物, 分析理解街景图像中景物的组成结构; 然后, 依据小孔成像模型推导出景物的图像坐标和真实深度之间的 关系，从而计算出图像内地面区域的深度信息; 并根据景物与地面之间的相对位置关系和景物自身的特征变化估计 图像内其它景物的深度信息, 最终得到整幅街景图像的深度估计结果. 实验表明, 该方法得到的街景图像深度估计 结果能准确反映图像内各个景物在真实世界中的深度分布, 在效果上要优于其它的方法.
\end{abstract}

关键词: 特征提取; 景物分类; 小孔成像模型; 深度估计

中图分类号: TP391.4 文献标识码: A 文章编号: 1002-0446(2011)-02-0174-07

\section{Depth Estimation from a Single Still Image of Street Scene Based on Content Understanding}

\author{
LI Le, ZHANG Maojun, XIONG Zhihui, XU Wei \\ (College of Information Systems and Management, National University of Defense Technology, Changsha 410073, China)
}

\begin{abstract}
A method for depth estimation by understanding how the objects compose the whole scene in a single image of street scene is presented. Firstly, a single image of street scene is segmented into regions. The features of each region and the associated features of its neighbor area are extracted. And the regions are classified as types of object with features of each region by machine learning method, which shows how the image is made up of every object. Then, the depth of ground is estimated by the relationship between coordinate in image and depth in the real world of the same object which is deduced from pin-hole imaging model. And the depth of others in image is estimated by not only the relative position between the objects and ground but also the change of some features in objects. The depth map of image is produced at last. The experiment shows that our algorithm performs better than others and the result of depth estimation reflects the location of each object in the real world exactly.
\end{abstract}

Keywords: feature extraction; object classification; pin-hole imaging model; depth estimation

\section{1 引言 (Introduction)}

从 2 维图像中估计图像内所包含的各个景物对 象的深度信息、获取场景的 3 维结构, 是目前计算 机视觉研究领域中的热点问题，在机器人视觉、虚 拟城市建模等领域有着广泛的应用前景.

目前大多数关于图像深度估计的研究都是基于 多幅图像的, 它根据对极几何原理, 结合拍摄时相机 运动产生的视差信息来估计深度, 如 SFM (structure from motion, 运动恢复结构) 算法 ${ }^{[1]}$. 而对于单幅图 像, 由于无法获取景物目标的视差信息, 只能通过 图像自身的一些特征和先验知识来获得有限的线索 以完成深度估计, 因此具有较高的技术难度. 目前,
已有单幅图像的深度估计大多是基于一定假设条件 的. 例如, Hertzmann ${ }^{[2]}$ 的方法必须已知图像中某个 景物对象的具体信息才能重建整个 3 维场景. 张大 志 ${ }^{[3]}$ 将图像场景看做不同深度层的集合, 通过贝叶 斯方法估计各层的深度信息, 完成对复杂场景的深 度估计. 人们通过分析图像中景物的成像质量发现， 图像拍摄时, 位于相机焦平面上的景物成像清晰, 而 远离焦平面的景物所成的像则较模糊, 通过分析图 像内景物目标的模糊程度可以估计每个景物的深度 信息 ${ }^{[4-5]}$. Saxena ${ }^{[6]}$ 根据景物对象在不同深度上所成 的像在纹理、阴影等方面的差异和景物之间的遮挡 关系等单目视觉中能反映深度关系的特征, 通过计 
算不同尺度下邻域像素特征的相关性, 使用结合了 高斯和拉普拉斯算子的 MRF (马尔可夫随机场) 模 型来综合估计每个像素的深度信息. 这些方法虽然 可以得到较为准确的深度信息, 但是计算复杂耗时, 而且每更换一个新的场景, 原有的模型将失效, 需 要重新采集相应的深度数据进行训练.

Hoiem $^{[7-9]}$ 在假设图像场景内的所有景物对象 都是垂直直立于地面的基础上, 提出一套完整的单 幅图像深度估计方法. 该方法以像素块为单位提取 颜色、纹理等特征, 然后使用 adboost 分类方法将 整个图像分解为地面、天空、直立景物等, 最后对 图像中的地面区域建立 3 维模型, 并依据图像中景 物对象和地面的相交位置重构出整个 3 维场景. 但 是, Hoiem 的深度估计方法存在以下不足: (1) 深度 估计是基于图像分类结果的, 而图像中景物目标的 识别率有待提高; (2) 所有景物的深度都是以地面为 参考的, 而地面区域的深度估计不够精确, 存在较 大误差; (3) 仅仅根据景物和地面的相交位置估计景 物深度, 没有体现景物自身的深度变化; (4) 对于景 物下方不直接和地面相交的情况无法给出合理的深 度估计结果。

为了解决 Hoiem 深度估计方法的上述缺陷, 本 文通过分析理解街景图像内景物之间的构图关系, 依据其中蕴含的深度认知线索估计街景图像的深度 信息. 算法流程如图 1 所示, 可分为内容理解和深 度估计两部分: (1) 理解街景图像的景物构成, 对图 像进行分块并提取特征, 通过机器学习的方法依据 图像块特征识别各类景物目标, 获取图像内各个景 物之间的构图关系; (2) 场景深度估计, 根据相机小 孔成像模型推导地面区域的深度和图像坐标之间的 对应关系, 完成地面区域的深度估计, 然后依据景 物与地面以及景物之间的构图关系估计场景内各个 景物的深度信息. 与 Hoiem 深度估计方法相比, 本 文的方法具有以下几个优点: (1) 使用邻域图像块联 合特征, 体现了景物内部各组成要素以及图像内相 邻景物之间的构图关系，从而提高景物目标分类识 别的准确率; (2) 依据相机小孔成像模型, 推导出图 像坐标和深度之间的对应关系, 从而减少深度估计 误差; (3) 对于那些无法直接参考地面进行深度估计 的景物, 则通过获取景物中部分区域的真实尺寸等 先验知识估计其深度, 或者依据景物之间的构图关 系进行深度分层, 从而体现景物在深度分布上的差 异; (4) 结合摄影测量方法, 推知建筑物的深度变化 趋势, 进一步估计建筑物的深度. 经过实验对比, 使 用本文方法对单幅街景图像进行深度估计, 各个景
物对象的深度信息更加准确、细致, 在效果上优于 Hoiem 方法.

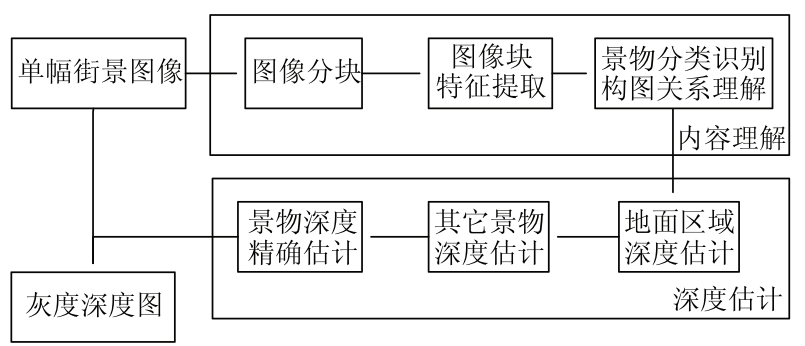

图 1 街景图像深度估计算法原理

Fig.1 Framework of depth estimation from image of street scene

\section{2 图像内容理解 (Understanding of image content)}

图像的内容理解主要是依据景物特征从街景图 像中识别每个景物目标, 从而解析图像内的景物结 构关系, 为后续的深度估计做准备.

\section{1 图像分块}

一幅图像是由许多个具有 RGB 颜色信息的像 素点所构成的 2 维阵列. 将图像内相似的像素点聚 合成图像块, 可以有效屏蔽底层像素特征的差异性 和复杂性, 有助于理解图像内容. 本文使用了基于 图论的方法进行图像分块 ${ }^{[10]}$. 首先将街景图像划 分为若干规则的像素块, 并构造无向图 $G=(V, E)$ 来进行描述, $V$ 表示图像的每一个像素块, $E$ 则表 示像素块之间的相邻关系 $\omega\left(v_{i}, v_{j}\right)$. 通过对像素块 $V$ 的合并聚类得到图像分块 $C$, 并建立相应的数 学模型来描述图像块 $C$ 内部各像素之间的一致性 $f_{\mathrm{Int}}(C)=\max _{v_{i}, v_{j} \in C} \omega\left(v_{i}, v_{j}\right)$ 和相邻图像块 $C_{1} 、 C_{2}$ 之间的 差异性 $f_{\mathrm{Dif}}\left(C_{1}, C_{2}\right)=\min _{v_{i} \in C_{1}, v_{j} \in C_{2}} \omega\left(v_{i}, v_{j}\right)$. 在初始状态, 每个像素块 $V$ 都是一个图像分块 $C$, 通过比较相邻 图像块 $C_{1} 、 C_{2}$ 之间的一致性和差异性, 动态迭代优 化对图像块合并聚类, 直至获得最优的图像分块结 果.

\section{2 图像块特征提取}

每类景物都有独特的特征, 通过提取每个图像 块自身的特征以及相邻图像块之间的相关特征, 为 判断图像块所属的景物类别提供依据.

- 图像块自身的特征

颜色可以很直观地描述图像块的特征, 现实生 活中的很多景物对象都具有非常典型的颜色特征. 例如, 天空总是蓝色或白色的, 且亮度很高.

位置和形状特征也为识别景物目标提供了重要 线索. 在图像中, 天空一般是位于图像的上方且对 
应的图像块具有较大的面积, 而地面则位于图像的 下方.

纹理特征反映了图像块内部像素的复杂性和变 化规律, 也是图像识别中的重要特征. 比如, 树上大 量的叶子交错掩映与万里无云的天空就是两种完全 不同的纹理.

- 邻域图像块的联合特征

在图像分块时, 不可能将每个对象都恰好分割 成一个图像块, 必然出现过分割的情况. 那么, 具有 相似的颜色、纹理等特征的相邻图像块很可能属于 同一个景物. 而对于特征不相似的相邻图像块, 或 者反映了同一对象内的各个组成要素之间的关系， 例如汽车是由车轮、车身、车窗 3 类不同特征的图 像块依据一定的位置关系组成的; 或者反映了图像 内景物的组成结构, 比如街景图像中天空一般不和 地面相邻、汽车位于地面附近等线索. 因此, 每个图 像块都与其邻域图像块的特征存在一定关系, 在提 取图像块特征时, 本文通过条件随机场 (CRF) 模型 [11] 对邻域图像块之间的特征相关性进行描述.

$$
P\left(C \mid x_{i}, \theta\right)=\frac{1}{Z} \exp \left(\prod_{x_{j} \in S, \theta_{k} \in \Psi} P\left(C \mid g_{i j}\left(x_{i}, x_{j}, \theta_{k}\right)\right)\right)
$$

式 (1) 中, 景物类别为 $C$, 图像块 $x_{i}$ 的邻域为 $S$, 特 征集 $\Psi$ 中的各类特征为 $\theta_{k}$, 函数 $g_{i j}$ 表示相邻图像 块 $x_{i}$ 和 $x_{j}$ 之间在特征上的相互影响关系. 这里, 使 用机器学习的方法通过对训练样本中的街景图像进 行学习训练得到式 (1) 中的参数, 然后根据式 (1) 可 以计算图像块 $x_{i}$ 在其邻域 $S$ 的各类特征作用下属于 景物 $C$ 的概率, 即邻域联合特征.

\section{3 图像块分类识别}

根据提取的图像块特征, 本文使用基于统计学 习理论的 SVM (support vector machine, 支持向量 机) 分类器 ${ }^{[12]}$ 对图像内容进行分类识别. SVM 的 基本原理是: 通过学习训练, 在多个不同类型的样 本 $\left(x_{i}, y_{i}\right)$ 之间, 寻找一个最优的分类面, 使得该分 类面不仅能将各类样本分开，而且分类间隔最大， 从而获得最佳的分类效果. 具体的实验步骤如下:

(1) 对街景图像进行分块, 并构建每个图像块 $x_{i}$ 的特征向量. 一方面依据图像块 $x_{i}$ 自身的颜色、位 置、纹理等特征构建特征向量; 另一方面考察图像 块 $x_{i}$ 与邻域图像块在各类特征之间的相关性, 即根 据式 (1) 计算图像块 $x_{i}$ 在其邻域各类特征作用下属 于景物 $C$ 的概率, 并以此来组织图像块邻域特征向 量.

(2) 使用训练样本中街景图像的景物特征, 采
用交叉验证的方法选择合适的惩罚因子和核函数参 数, 完成对 SVM 分类器的训练.

(3) 将待分类图像中每个图像块对应的特征向 量输入 SVM 分类器, 判断该图像块所属的景物类 别, 得到街景图像的分类识别结果.

(4) 根据图像中景物目标分类的结果, 确定每个 景物的初始轮廓曲线, 通过对 Snake 能量函数 ${ }^{[13]}$ 的 动态迭代优化来精确提取景物目标轮廓, 提高景物 分类识别的精度.

按照以上的方法, 依据街景图像中每个图像块 的特征, 使用 SVM 分类器判断该图像块所属的景 物类别, 从街景图像中识别各个景物目标, 获取景 物之间的相对位置关系, 理解街景图像的景物组成 结构, 如图 2.

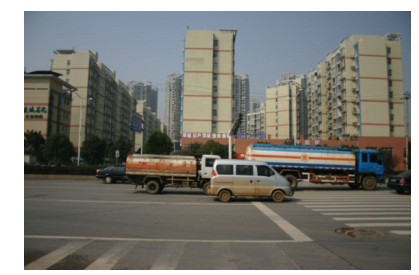

a 原始图像

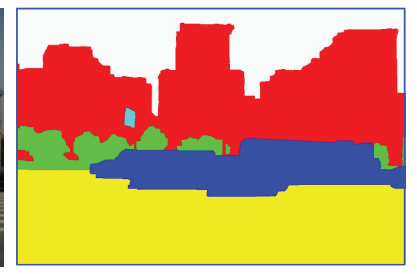

b 图像内容理解
白-天空 绿-植物 红-建筑物 蓝-汽车 黄-地面 青-路牌

图 2 图像内容理解

Fig.2 The result of understanding of image content

\section{3 图像深度估计 (Depth estimation from im- age )}

结合人的深度认知, 通过分析理解图像内景物 之间的构图关系, 首先估计地面区域的深度, 并以 地面的深度为参考, 根据景物和地面的相对位置以 及景物之间的前后遮挡关系, 依次确定每个景物的 深度信息.

\section{1 天空}

在真实世界中, 天空与相机拍摄位置之间的距 离远大于其它景物目标, 可以近似认为是无限远的, 因此相对于其它景物, 天空的深度可认为是最大的. 在处理图像中的天空区域时, 可以直接在灰度深度 图中将其深度指定为最大值.

\section{2 地面}

地面区域的深度估计是整幅图像深度估计的基 础, 通过分析相机的小孔成像模型, 可以估计地面 区域的深度信息.

在理想条件下, 认为相机成像模型符合小孔成 像模型, 因此本文依据相机小孔成像模型推导出真 实世界中地面点 $G$ 的深度和它在相机中所成像点 $g$ 的图像坐标之间的对应关系. 对于大多数的街景图 
像, 真实景物的深度 (即物距) 要远大于景物所成像 的像距, 根据凸透镜成像原理, 可近似认为景物所 成的像都位于相机焦平面上. 基于上面的假设, 可 以推理景物深度和所成像之间的对应关系.

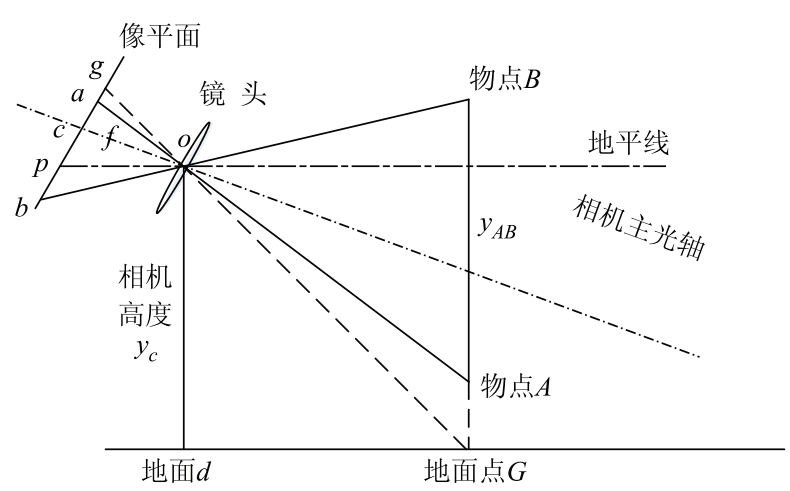

图 3 小孔成像模型

Fig.3 Pin-hole imaging model

如图 3 所示, 根据小孔成像模型, 所有的光线 均经过相机镜头的光心 $o$ 点, 主光轴与地面倾斜角 为 $\angle c o p$, 像平面中心点 $c$ 坐标为 $v_{c}$, 地面点 $G$ 所成 的像点 $g$ 的坐标为 $v_{g}$, 图像中地平线上点 $p$ 的坐标 为 $v_{p}$, 且相机感光 CCD (charge coupled device, 电 荷耦合器) 器件的实际尺寸与图像像素坐标之间的 比例关系为 $s$, 那么可以得到如下的关系:

$$
\begin{aligned}
\arctan \frac{y_{c}}{d} & =\arctan \frac{s\left(v_{g}-v_{c}\right)}{f}+\arctan \frac{s\left(v_{c}-v_{p}\right)}{f} \\
d & =\frac{y_{c}\left(f^{2}-s^{2}\left(v_{g}-v_{c}\right)\left(v_{c}-v_{p}\right)\right)}{f s\left(v_{g}-v_{p}\right)}
\end{aligned}
$$

式 (2) 反映了景物深度和所成像之间的对应关系, 其中 $f$ 和 $s$ 可以根据街景图像文件属性中记录的相 机拍摄参数获得, 而相机高度 $y_{c}$ 则由于街景图像一 般是由摄影师手持相机进行拍摄的, 可近似地认为 $y_{c}$ 为 $1.5 \mathrm{~m}^{[9]}$. 首先通过计算街景图像中的灭点、天 地边缘特征等方法分析估计街景图像内的地平线位 置 ${ }^{[14-15]}$, 获取地平线对应的坐标 $v_{p}$, 然后结合像平 面中心坐标 $v_{c}$, 计算地面区域像素点 $G$ 的深度信息. 通过实验分析, 发现该公式也适用于拍摄时相机旋 转所导致图像中地平线不水平的情况. 此时, 需要 依据图像的灭点等特征估计图像中地平线的位置, 并旋转图像将地平线调至水平, 再根据旋转之后地 面区域像素点的坐标使用式 (2) 计算图像中地面区 域的深度, 完成对街景图像地面区域的深度估计。

\section{3 其它景物}

- 根据景物与地面相对位置估计深度

按照人的深度认知, 街景图像中的汽车、建筑 物等景物都是直立于地面之上的. 因此, 在获得图
像中地面区域的深度之后, 以图像中地面区域作为 深度参考面, 依据景物与地面之间的相对位置关系, 在景物正下方检测提取其与地面的交线 $l_{c}$. 然后, 根 据深度估计式 (2), 按照交线 $l_{c}$ 上每个像素点的图像 坐标可以计算景物与地面相交位置 $l_{c}$ 的深度, 从而 确定该景物目标的深度信息.

- 景物与地面相对位置缺失时的深度估计

由于遮挡和成像范围的限制, 拍摄的图片中可 能存在某些景物与地面的相对位置关系无法确定的 情况, 此时无法通过地面来估计景物的深度信息. 但是, 如果事先知道景物本身或者其中一部分 (如 建筑物的窗户）的真实尺寸, 那么根据相机的小孔 成像模型, 通过比较景物目标的真实尺寸和对应的 像的尺寸，就可以估计出该景物的深度信息.

如图 3, 景物 $A B$ 的深度为 $d$, 其真实尺寸为 $y_{A B}$, 已知物点 $A$ 和 $B$ 分别成像于像平面的 $v_{a} v_{b}$ 处, 那 么根据三角函数的比例关系, 即 $y_{A B}=d(\tan \angle p o b+$ $\tan (\angle p o c+\angle a o c))$, 可以推导出景物的实际尺寸、像 的尺寸和深度之间的关系:

$$
\begin{aligned}
y_{A B}= & d \times\left(\frac{f s\left(v_{p}-v_{b}\right)}{f^{2}+s^{2}\left(v_{c}-v_{b}\right)\left(v_{c}-v_{p}\right)}+\right. \\
& \left.\frac{f s\left(v_{a}-v_{p}\right)}{f^{2}+s^{2}\left(v_{a}-v_{c}\right)\left(v_{c}-v_{p}\right)}\right)
\end{aligned}
$$

如果已知景物的真实尺寸 $y_{A B}$, 根据式 (3), 通 过比较景物的真实尺寸 $y_{A B}$ 和像的尺寸 $v_{a}-v_{b}$, 可 以估计该景物对应的深度 $d$.

但是, 对于某些特征不明显的景物, 可能无法 根据先验知识获取景物某部分的真实尺寸. 此时, 仅仅通过单幅图像中所蕴含的深度视觉线索无法正 确地估计景物的深度信息, 只能结合该景物与其周 围其它景物之间的遮挡关系来估计其深度. 对于此 种情况, 在估计景物深度时, 为了体现图像中不同 景物之间的深度差异, 使整幅图像的深度更具层次 感, 本文引入了深度分层参数 $L$. 通过分析图像内 景物 $X$ 与其邻近景物之间的构图关系, 找出在深度 上与景物 $X$ 相邻的景物目标 $Y$, 然后根据景物 $X$ 和 景物 $Y$ 之间的前后遮挡关系在景物 $Y$ 的深度上添加 或减去深度分层参数 $L$, 得到景物 $X$ 的深度, 以区 分景物 $X$ 和它邻近的景物 $Y$ 是处于不同的深度层之 上.

$$
d_{X}=d_{Y} \pm L
$$

通过使用大量的街景图像进行实验对比, 我 们发现将图像的总深度分为 25 个层次时 (即 $L=$ $255 / 25$ ), 人眼在前后两个相邻深度层的景物对象之 间就可以感觉到十分明显的深度层次差异. 
- 景物自身深度变化趋势的估计

在街景图像中, 对于建筑物这种大尺寸的景 物目标, 其自身的深度可能会出现较大的变化. 而 Hoiem 只是按照景物与地面的相对位置确定每个景 物的深度, 当建筑物的正面沿街分布时, 得到的景 物深度基本正确. 但是, 在街景图像中, 很多建筑物 的走向并不是沿街的, 靠近街边地面处的部分和远 离街边的部分在深度上完全不同. 为了获得准确的 景物深度信息, 需要结合摄影测量原理, 估计每个 建筑物在深度方向上的变化趋势.

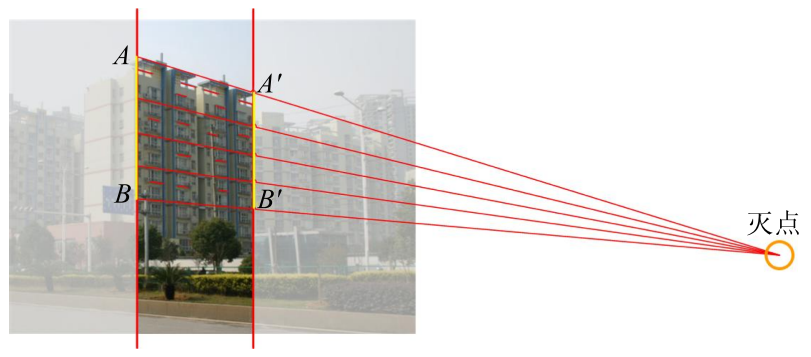

图 4 摄影测量原理

Fig.4 Photogram metric theory

对于一幅街景图像, 首先使用 Hough 变换 [16] 提取建筑物对象中的直线, 根据摄影测量理论, 将 建筑物对象中提取的直线延长寻找这些直线的交 点 (即灭点) ${ }^{[14]}$, 并结合建筑物的颜色、边缘等特 征从建筑物区域中区分出每栋建筑物的边界及其 走势 (图 4 中的 $A B$ 和 $A^{\prime} B^{\prime}$ ). 根据建筑物和地面之 间的相对位置关系, 可以确定建筑物靠近街边部分 的深度 $d_{A B}$ ( $A B$ 处的深度) 等于其正下方地面的深 度, 并且由于真实世界中该建筑物目标的边缘 $A B$ 和 $A^{\prime} B^{\prime}$ 具有同样的尺寸 (即 $y_{A B}=y_{A^{\prime} B^{\prime}}$ ), 因此根据 式 (3) 可以推导图像中景物特征的变化趋势和深度 之间的关系:

$$
\begin{aligned}
& \frac{\frac{d_{A^{\prime} B^{\prime}}}{d_{A B}}=}{\frac{v_{p}-v_{B}}{f^{2}+s^{2}\left(v_{c}-v_{B}\right)\left(v_{c}-v_{p}\right)}+\frac{v_{A}-v_{p}}{v_{p}-v_{B^{\prime}}}+s^{2}\left(v_{A}-v_{c}\right)\left(v_{c}-v_{p}\right)} \\
& \frac{v_{A^{\prime}}-v_{p}}{f^{2}+s^{2}\left(v_{c}-v_{B^{\prime}}\right)\left(v_{c}-v_{p}\right)}+\frac{s^{2}+s^{2}\left(v_{A^{\prime}}-v_{c}\right)\left(v_{c}-v_{p}\right)}{f^{2}}
\end{aligned}
$$

根据式 (5), 通过估计图像中建筑物目标的变化 趋势可以计算该建筑物对象内部每个像素点对应的 深度信息, 从而得到景物自身的深度变化, 使得景 物目标的深度估计结果能够反映其在真实世界中的 深度分布.

\section{4 实验结果 (Experimental results)}

实验中，使用佳能 300D 相机拍摄了 400 张分 辨率为 $800 \times 533$ 的街景图像作为实验图像库, 然后
从中选取了 200 幅作为训练图像集, 100 幅作为待 分类和深度估计的图像集. 并将街景图像中的景物 对象划分为天空、地面、建筑物、汽车等景物对象, 结合各个景物之间的相对位置关系估计街景图像的 深度.

本文的深度估计算法是建立在对图像内容理 解分类的基础上的, 关键是依据图像块的特征使用 SVM 分类器准确识别图像内每个景物对象. 本文首 先对街景图像进行分块, 以图像块为单位进行处理; 然后一方面使用颜色、形状等特征来描述每个图像 块自身的特性, 另一方面根据式 (1) 考察图像块邻 域特征之间的相关性用以描述图像块邻域的联合特 征, 最后通过 SVM 分类器识别街景图像内的各个 景物. 相比于传统的景物分类方法, 本文引入了图 像块邻域联合特征, 从而较好地反映景物内部以及 景物之间的构图关系, 取得了满意的景物目标识别 结果. 如表 1 所示, 本文的景物分类识别结果具有 较高的准确率, 尤其对街景图像中的天空、地面等 景物分类识别的准确率达到了 $90 \%$ 以上, 而对于建 筑物、汽车等结构复杂不易识别的景物准确率也达 到 $80 \%$, 保证了街景图像内容理解的准确性和可靠 性.

表 1 街景图像分类识别准确率

Tab.1 Classification accuracy of street scene image

\begin{tabular}{ccccc}
\hline 类别 & 天空 & 地面 & 建筑物 & 汽车 \\
准确率 & $95.7 \%$ & $93.3 \%$ & $79.5 \%$ & $81.7 \%$ \\
\hline 类别 & 树木 & 路牌 & 行人 & 平均 \\
准确率 & $85.8 \%$ & $87.0 \%$ & $71.3 \%$ & $85.2 \%$ \\
\hline
\end{tabular}

Hoiem 的图像分类实验中 [9], 先将景物分成天 空、地面、直立物体 3 类 (main class), 然后再进一 步识别具体的景物 (sub class), 如将直立物体分解 为汽车、建筑物等景物; 而本文的景物分类识别方 法, 使用了多种景物特征, 对各类景物的识别都取 得了较好的结果. 如表 2 所示, 本文的图像分类方 法无论是将街景图像分解为天空、地面、直立景物, 还是直接从街景图像中识别出汽车、行人等具体的

表 2 图像分类识别准确率比较

Tab.2 Comparison of image classification accuracy

\begin{tabular}{ccccc}
\hline & main class & sub class & 行人 & 汽车 \\
\hline Dalal-Triggs 2005 (Initial) & $\backslash$ & $\backslash$ & $54 \%$ & $41 \%$ \\
Hoiem et al. 2006 (Iter 1) & $\backslash$ & $\backslash$ & $66 \%$ & $41 \%$ \\
Hoiem.2008 (Contextual) & $87.6 \%$ & $61.8 \%$ & $61 \%$ & $51 \%$ \\
本文的方法 & $92.6 \%$ & $85.2 \%$ & $71.3 \%$ & $81.7 \%$ \\
\hline
\end{tabular}



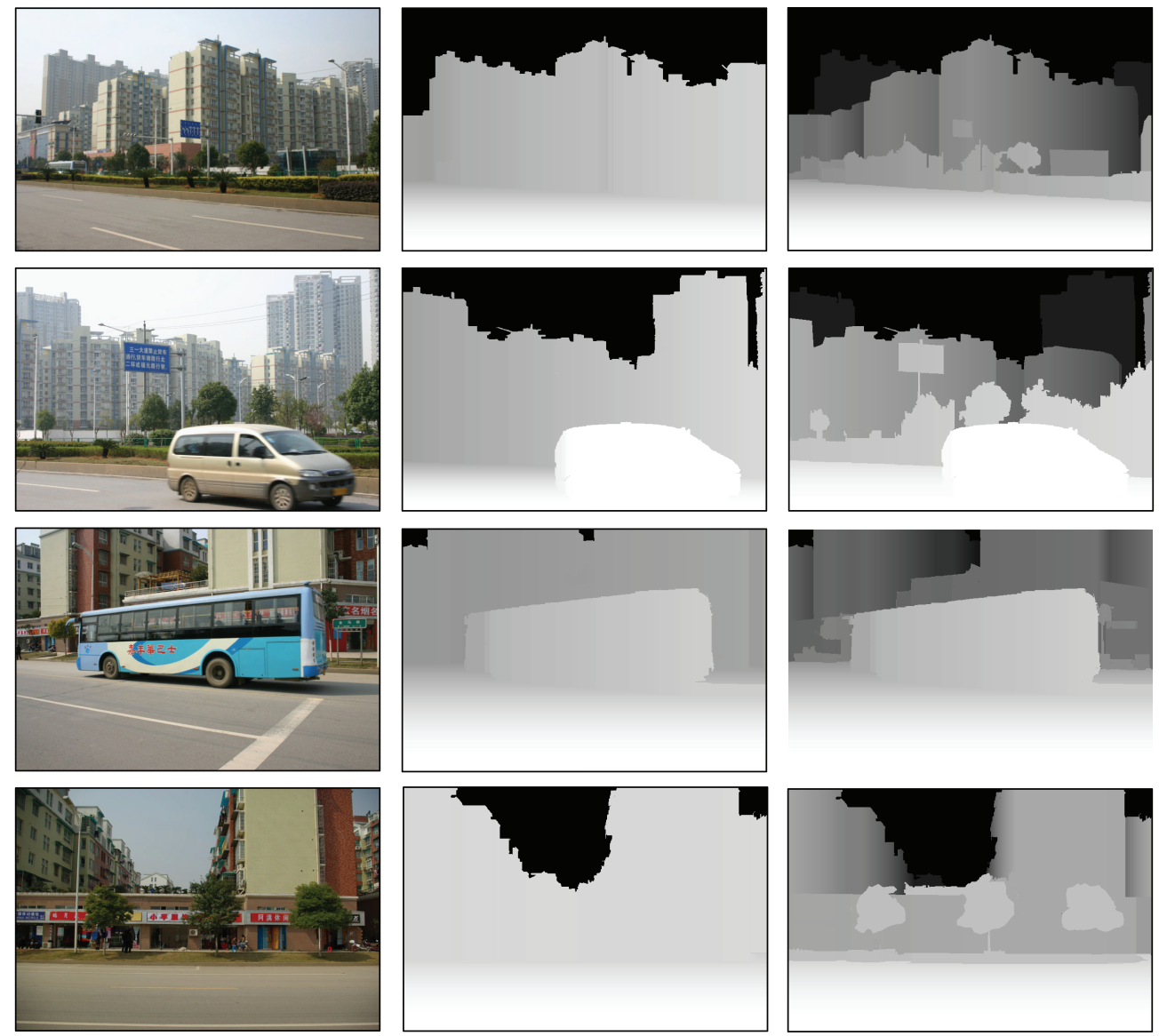

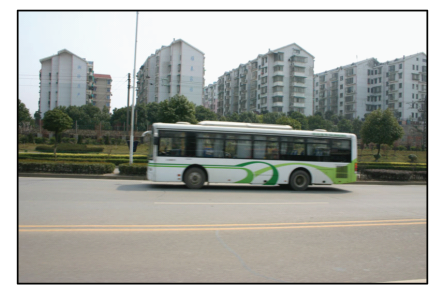

(a) 原始图像

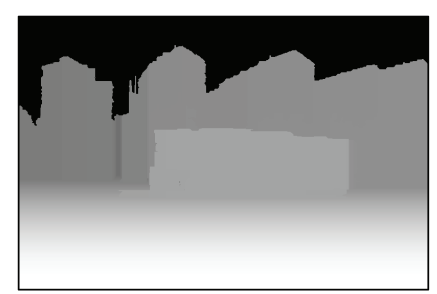

(b) Hoiem的方法

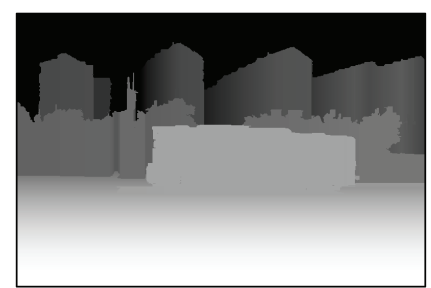

(c) 本文的方法

图 5 街景图像深度估计实验结果

Fig.5 The results of depth estimation from street scene image

景物目标, 本文的方法在景物分类识别的准确率上 要明显优于其他人的方法.

完成对街景图像的分类识别, 解析出图像场景 的景物构成之后, 根据式 (2) 首先估计图像中地面 区域的深度, 并依据景物与地面以及景物之间的相 对位置关系, 估计每个景物的深度信息. Hoiem 的 深度估计方法中, 在得到地面区域的深度之后, 只 是根据景物与地面的邻接位置做了一个 “pop up” 的 操作, 对于景物下方不直接和地面相接, 以及景物 一侧邻街、一侧远离街边的情况, 无法给出正确的 深度估计结果. 而本文则针对街景图像中这些情况, 给出了相应的处理方法. 对于不直接和地面相交、 无法根据地面来估计深度的景物目标, 通过获取的 景物目标真实尺寸等先验知识, 结合式 (3) 实现景 物深度估计; 如果无法知晓这些先验知识, 那么依
据景物之间的前后遮挡关系，使用深度分层参数以 区分景物之间的前后深度差异. 此外, 对于街景图 像中的建筑物目标, 使用摄影测量的方法, 估计建 筑物的走向, 从而根据式 (5) 获得建筑物中不同位 置的深度信息, 使得街景图像的深度估计结果更加 细致、准确. 如图 5 所示, 本文对单幅街景图像的估 计结果中, 建筑物、植物、汽车等景物目标之间具有 明显的前后深度层次关系, 而且较准确地反映出建 筑物自身的深度变化, 在效果上要优于 Hoiem 的方 法.

\section{5 结论 (Conclusion)}

本文针对单幅街景图像的特点提出了一套完整 的深度估计方法. 通过对单幅图像分块并提取景物 特征, 实现了对图像内容的分类识别, 获取图像中 
景物对象之间的构图关系; 然后根据相机小孔成像 模型精确估计地面区域的深度, 并依据景物与地面 的相对位置以及景物之间的遮挡关系估计每个景 物的深度信息. 与 Hoiem 的深度估计结果相比, 本 文的深度估计结果能够更加真实地反映街景图像内 各个景物的深度分布关系. 目前, 我们仅完成了街 景图像的深度估计, 在今后的工作中, 需要进一步 研究改进图像特征提取和景物识别分类的算法, 拓 展景物的识别范围, 实现对更多种类图像的深度估 计.

\section{参考文献（References）}

[1] Forsyth D A, Ponce J. Computer vision: A modern approach [M]. Englewood Cliffs, NJ, USA: Prentice Hall, 2003.

[2] Hertzmann A, Seitz S M. Example-based photometric stereo: Shape reconstruction with general, varying BRDFs[J]. IEEE Transactions on Pattern Analysis and Machine Intelligence, 2005, 27(8): 1254-1264.

[3] 张大志, 王勇涛, 田金文, 等. 基于单目视觉系统的远距离 场景重建算法研究 [J]. 宇航学报, 2008, 29(1): 289-294.

Zhang D Z, Wang Y T, Tian J W, et al. Efficient 3D reconstruction using monocular vision[J]. Journal of Astronautics, 2008, 29(1): 289-294.

[4] Ens J, Lawrence P. An investigation of methods for determining depth from focus[J]. IEEE Transactions on Pattern Analysis and Machine Intelligence, 1993, 15(2): 97-108.

[5] 曾祥尽, 黄心汉, 吴倩, 等. 马尔可夫随机场在显微图像散 焦深度信息估计中的应用 [J]. 机器人, 2008, 30(5): 416420 .

Zeng X J, Huang X H, Wu Q, et al. Application of MRF to depth information estimation of micro image defocus[J]. Robot, 2008, 30(5): 416-420.

[6] Saxena A, Chung S H, Ng A Y. 3-D depth reconstruction from a single still image[J]. Computer Vision, 2008, 76(1): 53-69.

[7] Hoiem D, Efros A A, Hebert M. Automatic photo popup[C]//32nd International Conference on Computer Graphics and Interactive Techniques. 2005: 577-584.
[8] Hoiem D, Efros A A, Hebert M. Closing the loop on scene interpretation[C]//IEEE Conference on Computer Vision and Pattern Recognition. Piscataway, NJ, USA: IEEE, 2008: 1-8.

[9] Hoiem D, Efros A A, Hebert M. Putting objects in perspective[C]//IEEE Conference on Computer Vision and Pattern Recognition. Piscataway, NJ, USA: IEEE, 2006: 2137-2144.

[10] Felzenszwalb P, Huttenlocher D. Efficient graph-based image segmentation[J]. International Journal of Computer Vision, 2004, 59(2): 167-181.

[11] Shotton J, Winn J, Rother C, et al. TextonBoost: Joint appearance, shape and context modeling for multi-class object recognition and segmentation[C]/Europeon Conference on Computer Vision. Piscataway, NJ, USA: IEEE, 2006: 1-15.

[12] Chang C C, Lin C J. LIBSVM: A library for support vector machines[EB/OL]. (2010-04-01) [2010-05-10]. http://www. csie.ntu.tw/ cjlin/libsvm.

[13] Kass M, Wit K A, Terzopoulos D. Snakes: Active contour models[J]. International Journal of Computer Vision, 1988, 1(4): 321-331.

[14] Havasi L, Szirányi T. Extraction of horizontal vanishing line using shapes and statistical error propagation[C]//Photogrammetric Computer Vision Symposium of International Society for Photogrammetry and Remote Sensing Commission III. 2006: 167-173.

[15] Zafarifar B, Weda H, de With P H N. Horizon detection based on sky-color and edge features[C]//Proceedings of the SPIE, vol.6822. Bellingham, WA, USA: SPIE, 2008: 682220-6822209.

[16] Duda R, Hart P. Use of the Hough Transformation to Detect Lines and Curves in Pictures[J]. Communications of the ACM, 1972, 15(1): 11-15.

\section{作者简介:}

李 乐 (1983-), 男, 博士生. 研究领域: 多媒体信息系统 与虚拟现实技术, 机器视觉, 嵌入式系统设计.

张茂军 (1971-), 男, 教授, 博士生导师. 研究领域: 多媒 体信息系统与虚拟现实技术，视频图像处理.

熊志辉 (1976-), 男, 博士, 副教授. 研究领域: 视频图像 处理, 机器视觉和全向成像技术. 